\title{
On Kirchhoff's Law and Its Generalized Application to Absorption and Emission by Cavities*
}

\author{
Francis J. Kelly
}

(March 15, 1965)

\begin{abstract}
Several authors have made the assumption that Kirchhoff's Law holds for the apparent local spectral emittance and apparent local spectral absorptance of any point on the interior surface of a cavity. The correctness of this assumption is demonstrated under certain general conditions, and its practical application to the calculation of the total flux absorbed by a cavity or spacecraft is discussed. A further application to the case of a nonisothermal cavity or spacecraft is derived. By this derivation an easy method for determining the total flux emitted from such a nonisothermal cavity is found when the distribution of the apparent local spectral emittance of the isothermal cavity is known. The economy and versatility of this method is shown by the calculation of the total flux emitted from a nonisothermal cylindrical cavity for several arbitrary cases of temperature distribution on the interior surface of the cavity. Finally, the integral equation for a diffuse cavity whose wall emittance varies with position on the wall is transformed to an equation having a symmetric kernel.
\end{abstract}

\section{Introduction}

Kirchhoff's Law states that at a point on the surface of a thermal radiator at any temperature and wavelength, the spectral directional emittance is equal to the spectral absorptance for radiation incident from the same direction. ${ }^{1}$ This law, coupled with the law of conservation of energy, permits us to relate the emittance, absorptance, and reflectance at a point on the surface of an opaque thermal radiator by eq (1).

$$
\boldsymbol{\epsilon}(\lambda)=\alpha(\lambda)=1-\rho(\lambda)
$$

This relation has been used by Wien and Lummer, Ribaud, Gouffé, Michaud, and Page (refs. $\left.[1-5]^{2}\right)$, to derive expressions for the emittance of a cavity or enclosure with a finite opening. In these derivations the implicit assumption seems to be made (without explicit proof) that the apparent local spectral emittance at a point within an isothermal cavity is equal to the apparent local spectral absorptance at that same point. ${ }^{3}$ Although it is not immediately obvious that Kirchhoff's Law can be thus extended to these "apparent local" quantities, the analysis presented in section 2 based on the assumptions and techniques of diffuse interreflection theory shows that it does hold. The proof is purposely made for the general case, so as to be applicable to opaque isothermal cavities of any shape with one or more openings. It thus applies to any spacecraft configuration as a special case of a cavity. The emittance of the wall material is not assumed to be uniform over the cavity walls, but the emittance instead is permitted to vary with position within the cavity. The proof, however, is limited to a cavity with diffusely reflecting and emitting walls. Although a proof for the more general case of a a cavity with nondiffusely reflecting and emitting walls is not presented here, such a proof might possibly be developed by an approach similar to that of C. von Fragstein [6].

*This paper was presented at the AIAA Second Aerospace Sciences Meeting, New York, N.Y. on January 27, 1965 . This work was done under the sponsorship of the Marshall Space Flight Center of NASA and of the Air Force Materials Laboratory, Research and Technology Divisions, Air Force Systems Command.

${ }^{1}$ The spectral directional emittance is defined as the ratio of the flux per unit area, unit solid angle, and unit wavelength emitted at wavelength $\lambda$ in a particular direction $(\varphi, \theta)$ from a surface element to the radiant flux per unit area, unit solid angle, and unit wavelength emitted at wavelength $\lambda$ in the same direction $(\varphi, \theta)$ from the surface of a blackbody radiator at the same temperature.

${ }^{2}$ Figures in brackets indicate the literature references at the end of this paper.

${ }^{3}$ For the definitions of these "apparent local" quantities, see section 2. 
In section 3 the equations developed in section 2 are discussed, and they are shown to have a practical application to the problem of determining the radiant flux absorbed by a cavity. In section 4 a novel method is derived for determining the total flux emitted from a nonisothermal cavity, while in section 5 the method derived in section 4 is applied to some sample calculations. This is done by determining the heat flux from a cylindrical cavity for several different cases of temperature distribution within the cavity. Finally in the appendix the integral equation characteristic of a cavity with nonuniform wall emittance is transformed into an integral equation with a symmetric kernel.

\section{Proof of Kirchhoff's Law for a Diffuse Cavity}

First the general reflecting geometry of a cavity will be established. An enclosure $C$ of arbitrary shape which has one or more openings is considered throughout. Each point on the interior surface of the enclosure is identified with a coordinate vector, $\mathbf{x}$ in a convenient frame of reference. The coordinate vector, $\mathbf{x}$ is used here because some quantities will be thought of as functions of position on the interior surface, or equivalently, as functions of $\mathbf{x}$. It is assumed that the enclosure's walls are opaque and that they reflect and emit diffusely. It is further assumed that the material and temperature of the interior wall may vary as a function of position on the wall surface (that is as a function of $\mathbf{x}$ ), but that the emittances of the various wall materials are independent of temperature. The spectral emittance, absorptance, and reflectance of the wall material at the point $\mathbf{x}$ are denoted by $\epsilon(\lambda, \mathbf{x}), \alpha(\lambda, \mathbf{x})$ and $\rho(\lambda, \mathbf{x})$ respectively. In accordance with Kirchhoff's Law the wall material's emittance, absorptance, and reflectance at each point $\mathbf{x}$ are related to one another according to (2)

$$
\boldsymbol{\epsilon}(\lambda, \mathbf{x})=\alpha(\lambda, \mathbf{x})=1-\rho(\lambda, \mathbf{x})
$$

It is assumed that (2) is valid whatever the wavelength or angular distribution of flux falling on the surface. Thus all fluorescence and stimulated emission effects that might invalidate (2) are neglected.

Next, the presence of radiant flux within the cavity is considered. The symbol $B_{\lambda}(\mathbf{x})$ stands for the spectral radiosity or the radiant flux per unit area and unit wavelength leaving an element $d A(\mathbf{x})$ on the cavity interior. The symbol $E_{\lambda}(\mathbf{x})$ stands for the spectral irradiance or the radiant flux per unit area and unit wavelength incident on $d A(\mathbf{x})$. The radiosity is given by

$$
B_{\lambda}(\mathbf{x})=\epsilon(\lambda, \mathbf{x}) B_{\lambda, b}[T(\mathbf{x})]+[1-\epsilon(\lambda, \mathbf{x})] E_{\lambda}(\mathbf{x})
$$

where $B_{\lambda, b}[T(\mathbf{x})]$ stands for the flux per unit area and unit wavelength emitted from a blackbody radiator at temperature $T(\mathbf{x})$ and wavelength $\lambda$. It is convenient for the purposes of this paper to divide $E_{\lambda}(\mathbf{x})$ into two parts: $E_{\lambda, e}(\mathbf{x})$, the external spectral irradiance is the flux per unit area and unit wavelength incident on $\mathbf{x}$ directly from a source outside the cavity; $E_{\lambda, c}(\mathbf{x})$, the cavity spectral irradiance is the spectral flux per unit area and unit wavelength incident on $\mathbf{x}$ whose source is the radiosity distribution $B_{\lambda}\left(\mathbf{x}_{0}\right)$ at all points $\mathbf{x}_{0}$ within the cavity. $E_{\lambda}(\mathbf{x})$ is given by

$$
E_{\lambda}(\mathbf{x})=E_{\lambda, e}(\mathbf{x})+E_{\lambda, c}(\mathbf{x})
$$

The cavity irradiance at a point $\mathbf{x}, E_{\lambda, c}(\mathbf{x})$, is related to the radiosity, $B_{\lambda}\left(\mathbf{x}_{0}\right)$, of every point $\mathbf{x}_{0}$ according to

$$
E_{\lambda, c}(\mathbf{x})=\int_{c} B_{\lambda}\left(\mathbf{x}_{0}\right) K\left(\mathbf{x}_{0}, \mathbf{x}\right) d A\left(\mathbf{x}_{0}\right)
$$

where $K\left(\mathbf{x}_{0}, \mathbf{x}\right)$ is the diffuse angle factor or configuration factor which gives the irradiance at $\mathbf{x}$ 
caused by a unit area of a diffuse source located at $\mathbf{x}_{0}$. The following important relation is obtained by combining (3), (4), and (5).

$$
B_{\lambda}(\mathbf{x})=\epsilon(\lambda, \mathbf{x}) B_{\lambda, b}[T(\mathbf{x})]+[1-\epsilon(\lambda, \mathbf{x})] E_{\lambda, e}(\mathbf{x})+[1-\epsilon(\lambda, \mathbf{x})] \int_{c} B_{\lambda}\left(\mathbf{x}_{0}\right) K\left(\mathbf{x}_{0}, \mathbf{x}\right) d A\left(\mathbf{x}_{0}\right) .
$$

There is only one function $B_{\lambda}(\mathbf{x})$ which will "satisfy" this equation when the functions $T(\mathbf{x})$, $E_{\lambda, e}(\mathbf{x}), \epsilon(\lambda, \mathbf{x})$, and $K\left(\mathbf{x}_{0}, \mathbf{x}\right)$ are given. Equation (6) in which $B_{\lambda}(\mathbf{x})$ is the only unknown function, thus completely determines $B_{\lambda}(\mathbf{x})$. Many well-known methods exist for finding the solution, $B_{\lambda}(\mathbf{x})$, to such an integral equation, although in some cases the actual evaluation of $B_{\lambda}(\mathbf{x})$ may be somewhat tedious. The transformation of eq (6) into the form of a Fredholm integral equation of the second kind with a symmetric kernel, is shown in the appendix.

Next the apparent local spectral emittance inside an isothermal emitting cavity which is not irradiated by an outside source will be defined. For this circumstance $T(\mathbf{x})=T$ (a constant) and $E_{\lambda, e}(\mathbf{x})=0$ everywhere over the interior surface of the cavity. Then the apparent local spectral emittance $\epsilon_{a}(\lambda, \mathbf{x})$ is defined as

$$
\epsilon_{a}(\lambda, \mathbf{x}) \equiv \frac{B_{\lambda}(\mathbf{x})}{B_{\lambda, b}[T]}
$$

where $B_{\lambda}(\mathbf{x})$ is the radiosity distribution within the cavity. The integral equation which governs $\epsilon_{a}(\lambda, \mathbf{x})$ is obtained by substituting the above conditions in (6) and dividing both sides by $B_{\lambda, b}[T]$.

$$
\epsilon_{a}(\lambda, \mathbf{x})=\epsilon(\lambda, \mathbf{x})+[1-\epsilon(\lambda, \mathbf{x})] \int_{c} \epsilon_{a}\left(\lambda, \mathbf{x}_{0}\right) K\left(\mathbf{x}_{0}, \mathbf{x}\right) d A\left(\mathbf{x}_{0}\right) .
$$

Equation (8) completely determines the function $\epsilon_{a}(\lambda, \mathbf{x})$ as truly characteristic of the cavity because $\epsilon_{a}(\lambda, \mathbf{x})$ depends only on the cavity geometry and the emittance of the wall material.

Next, the apparent local spectral absorptance $\alpha_{a}(\lambda, \mathbf{x})$ is defined as a dimensionless function of position $\mathbf{x}$ such that if $Q_{\lambda, \alpha}$ is the total monochromatic flux absorbed within the cavity from any monochromatic external irradiance distribution $E_{\lambda, e}(\mathbf{x})$, then $Q_{\lambda, \alpha}$ may be calculated from

$$
Q_{\lambda, \alpha}=\int_{c} \alpha_{a}(\lambda, \mathbf{x}) E_{\lambda, e}(\mathbf{x}) d A(\mathbf{x}) .
$$

An equivalent definition of $\alpha_{a}(\lambda, \mathbf{x})$ is: $\alpha_{a}(\lambda, \mathbf{x})$ represents the dimensionless fraction of the monochromatic flux from outside the cavity incident on an element of area, $d A(\mathbf{x})$, that is absorbed within the cavity. This is illustrated in figure 1 for a focused incident beam, where a finite flux $Q_{\lambda, e}$ is shown striking the infinitesimal area $d A(\mathbf{x})$. For this focused beam, an amount $Q_{\lambda, e} \alpha(\lambda, \mathbf{x})$ is absorbed on the first reflection. The remainder is diffusely reflected both to the other parts of the cavity and to the cavity opening. The irradiance on the other points $\mathbf{x}_{0}$ within the cavity after this first reflection but before the second is given by (10).

$$
E_{\lambda, 2}\left(\mathbf{x}_{0}\right)=Q_{\lambda}[1-\alpha(\lambda, \mathbf{x})] K\left(\mathbf{x}, \mathbf{x}_{0}\right) .
$$

FIGURE 1. Focused beam incident at a point inside a cavity.

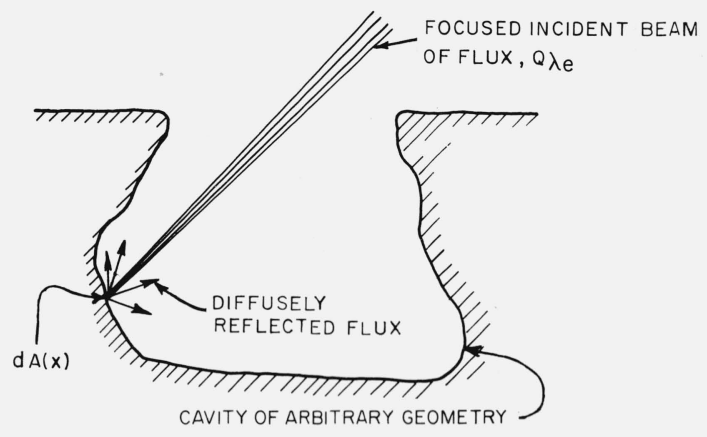


Since $E_{\lambda, 2}\left(\mathbf{x}_{0}\right)$ could be exactly simulated in principle by an irradiance from outside the cavity, the total flux absorbed by the cavity from $E_{\lambda},{ }_{2}\left(\mathbf{x}_{0}\right)$ may be calculated from (9). Thus we obtain (11) for the total flux absorbed.

$$
Q_{\lambda, \alpha}=Q_{\lambda, e} \alpha(\lambda, \mathbf{x})+Q_{\lambda, e}[1-\alpha(\lambda, \mathbf{x})] \int_{c} \alpha_{a}\left(\lambda, \mathbf{x}_{0}\right) K\left(\mathbf{x}, \mathbf{x}_{0}\right) d A\left(\mathbf{x}_{0}\right) .
$$

Because $\alpha(\lambda, \mathbf{x})=\epsilon(\lambda, \mathbf{x})$ from Kirchhoff's law, $K\left(\mathbf{x}, \mathbf{x}_{0}\right)=K\left(\mathbf{x}_{0}, \mathbf{x}\right)$ from angle factor algebra, and for the focused radiation considered, $\alpha_{a}(\lambda, \mathbf{x})=Q_{\lambda}, \alpha_{\alpha} Q_{\lambda}, e$ by definition, we obtain (12) for $\alpha_{a}(\lambda, \mathbf{x})$, which is equivalent to (8) for $\epsilon_{a}(\lambda, \mathbf{x})$.

$$
\alpha_{a}(\lambda, \mathbf{x})=\epsilon(\lambda, \mathbf{x})+[1-\epsilon(\lambda, \mathbf{x})] \int_{c} \alpha_{a}\left(\lambda, \mathbf{x}_{0}\right) K\left(\mathbf{x}, \mathbf{x}_{0}\right) d A\left(\mathbf{x}_{0}\right)
$$

Thus, it follows that

$$
\epsilon_{a}(\lambda, \mathbf{x})=\alpha_{a}(\lambda, \mathbf{x})
$$

i.e., the apparent local spectral emittance and apparent local spectral absorptance are equal, and Kirchhoff's law holds.

\section{Application to Cavity Âbsorption}

A direct consequence of (9) and (11) is that the solution of the integral equation governing the apparent local spectral emittance within a cavity also gives the information necessary for determining the total flux absorbed by the cavity for any given external irradiance. It is obvious that any conceivable spacecraft configuration is just a special case of the general geometric cavity configuration discussed in the first paragraph of section 2. If the spacecraft has diffusely reflecting surfaces, then $\epsilon_{a}(\lambda, \mathbf{x})$ for its particular configuration may be calculated by (8). The total flux absorbed by the spacecraft for any given external irradiance distribution (which would correspond to any given orientation of the spacecraft with respect to the sun, earth, moon or any other celestial object in the neighborhood) can be calculated easily by simply evaluating the integral in (9) based on the solution of (8). The different values of flux absorbed which correspond to the many different possible source positions with respect to the spacecraft can be evaluated quickly to any desired degree of accuracy by using modern computer techniques. This calculational procedure has actually been employed for certain cases of illumination in shallow cylindrical cavities and good agreement with experimental values was found [7].

\section{Application to Nonisothermal Cavity Emission}

Another application of the equation derived in section 2 is to the calculation of the total flux emitted through the opening of a nonisothermal heated cavity (or equivalently from the surface of a nonisothermal spacecraft). It is evident from (6) that both $\epsilon(\lambda, \mathbf{x}) B_{\lambda, b}[T(\mathbf{x})]$ and $[1-\epsilon(\lambda, \mathbf{x})]$ $E_{\lambda, e}(\mathbf{x})$ play the same mathematical role in determining $B_{\lambda}(\mathbf{x})$. As a consequence, both of the following cases will give rise to identical radiosity distributions within the cavity and fluxes leaving the cavity.

Case 1. Arbitrary temperature distribution $=T(\mathbf{x})$; external irradiance distribution $=E_{\lambda, e}(\mathbf{x})=0$, everywhere.

Case 2. Temperature distribution $=T^{\prime}(\mathbf{x})=0$, everywhere; external irradiance distribution $=E_{\lambda, e}^{\prime}(\mathbf{x})=\epsilon(\lambda, \mathbf{x})[1-\epsilon(\lambda, \mathbf{x})]^{-1} B_{\lambda, b}[T(\mathbf{x})]$.

This can easily be seen by substituting both Case 1 and 2 into (6) and noting that exactly the same equation results in both cases. In order to find the total flux emitted by the cavity in Case 1, 
it is necessary to find in Case 2 the difference between the total incident flux and the total absorbed flux. The total incident flux $Q_{\lambda, e}$, is simply given by (14).

$$
Q_{\lambda, e}=\int_{c} E_{\lambda, e}^{\prime}(\mathbf{x}) d A(\mathbf{x}) .
$$

The total absorbed flux $Q_{\lambda, \alpha}$ is given by (9). Hence the total flux emitted, $Q_{\lambda, \epsilon}$ is

$$
Q_{\lambda, \epsilon}=\int_{c}\left[1-\alpha_{a}(\lambda, \mathbf{x})\right] E_{\lambda, e}^{\prime}(\mathbf{x}) d A(\mathbf{x})
$$

or in terms of the arbitrary temperature distribution $T(\mathbf{x})$ and the apparent local spectral emittance $\epsilon_{a}(\lambda, \mathbf{x})$

$$
Q_{\lambda, \epsilon}=\int_{c}\left[1-\epsilon_{a}(\lambda, \mathbf{x})\right] \epsilon(\lambda, \mathbf{x})[1-\epsilon(\lambda, \mathbf{x})]^{-1} B_{\lambda, b}[T(\mathbf{x})] d A(\mathbf{x}) .
$$

Because $T(\mathbf{x})$ is completely arbitrary, and because no restrictions have been made on the possible geometry of the cavity (or spacecraft), eq (15b) applies to all situations.

\section{The Flux From a Cylindrical Cavity}

To illustrate the usefulness of (15b) the total heat flux from a cylindrical cavity has been calculated for several different temperature distributions (see fig. 2).

FIGURE 2. Arbitrary temperature distributions within a cylindrical cavity.

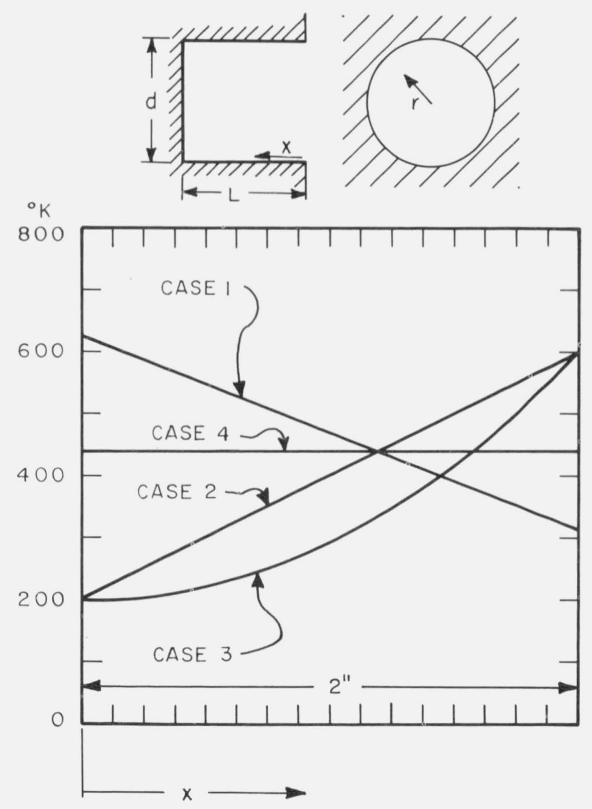

The apparent local spectral emittance $\epsilon_{a}(\lambda, \mathbf{x})$ has been published for a diffuse cylindrical cavity of uniform wall emittance, $\epsilon(\lambda)$ of 0.5 [8]. In the following calculations, these results are used to determine the total flux from an identical nonisothermal cavity. The cavity considered had a 1.0 in. radius and a $2.0 \mathrm{in.} \mathrm{depth.} \mathrm{Four} \mathrm{arbitrary} \mathrm{temperature} \mathrm{distributions} \mathrm{shown} \mathrm{in} \mathrm{figure}$ 2 were considered. These temperature distributions are assumed to be angularly symmetrical about the central axis of the cylinder. The base of the cavity is assumed to be isothermal with its temperature equal to the temperature at $x=2 \mathrm{in}$. in all four cases. These four temperature distributions were chosen to show that wide variations of temperature are easily handled by this method. Here $x$ is the distance from the mouth of the cavity along the walls; $r$ is the radial distance from the center of the cavity base. The apparent local spectral emittance distribution 
$\epsilon_{a}(\lambda, x)$ and $\epsilon_{a}(\lambda, r)$ were taken from reference [7], figure 4. For convenience, it is assumed that the cavity wall material is gray (its spectral emittance does not vary with wavelength). For this case the Stefan-Boltzmann law is applicable and $Q_{\lambda, \epsilon}, B_{\lambda, b}[T(\mathbf{x})], \epsilon(\lambda)$ and $\epsilon_{a}(\lambda, x)$ can be replaced by $Q_{\epsilon}, \sigma[T(\mathbf{x})]^{4}, \epsilon$, and $\epsilon_{a}(\mathbf{x})$ respectively. The total flux emitted from the mouth is given by (15c).

$$
Q_{\epsilon}=2 \pi \epsilon \sigma[1-\epsilon]^{-1}\left\{\frac{d}{2} \int_{0}^{L}\left[1-\epsilon_{a}(x)\right][T(x)]^{4} d x+\int_{0}^{d / 2}\left[1-\epsilon_{d}(r)\right][T(r)]^{4} r d r\right\} .
$$

The integrals in (15c) were approximated by Simpson's rule summations. Table 1 gives the total heat flux for each of the four cases. The first line of table 1 gives the results calculated with $\Delta x$ and $\Delta r$ equal to $0.1 \mathrm{in}$. The second line gives the results when $\Delta x$ and $\Delta r$ are taken to be $0.05 \mathrm{in}$. The results of the calculation based on the larger $\Delta x$ and $\Delta r$ agree to within 0.6 percent with those using the smaller $\Delta x$ and $\Delta r$. These results were obtained with relatively little expenditure of time and effort. For the three cases which have the same average temperature (1,2, and 4), the isothermal case emits the least flux, and the cavity whose temperature decreased from a maximum at the front emits the greatest flux. The values obtained for the (isothermal) case 4 can be directly compared to data given in [8]. Using $Q_{\epsilon} / \sigma T^{4}\left(\pi d^{2} / 4\right)=0.8084$ from table 2 of [8], one calculates $Q_{\epsilon}=3.484 \mathrm{~W}$, whereas the result from the integration in the present paper gives $Q_{\epsilon}=3.49370 \mathrm{~W}$. When $T(x)=T$ (a constant) everywhere within the cavity, eq (15c) of the present paper reduces to eq (1la) of [8].

TABLE 1. Q $\mathrm{Q}_{\epsilon}$ for cases 1 to 4

\begin{tabular}{|c|c|c|c|c|}
\hline & Case 1 & Case 2 & Case 3 & Case 4 \\
\hline$\Delta x=0.1 \mathrm{in} .$. & 5.94 & 4.269 & 3.348 & 3.49373 \\
\hline$\Delta x=0.05$ in $\ldots \ldots \ldots \ldots \ldots \ldots$ & 5.97 & 4.265 & 3.34 .6 & 3.49370 \\
\hline
\end{tabular}

The main limitation on the application of (15b) is the need for the function $\epsilon_{a}(\lambda, \mathbf{x})$ for various cavity (or spacecraft) geometries. The function $\epsilon_{a}(\lambda, \mathbf{x})$ has been determined for only a limited number of simple geometries like the sphere, the cone, and the cylinder. [9-11]. It is to be hoped that more $\epsilon_{a}(\lambda, \mathbf{x})$ data will appear in the future, because its knowledge permits the determination of the flux to and from a cavity under a great number of conditions.

The author thanks D. G. Moore, J. C. Richmond, and A. C. Kelly for their valuable assistance in the preparation of this paper; and Dr. E. M. Sparrow for a helpful conversation.

\section{Appendix}

Equation (8) may be transformed into the form of a Fredholm Integral equation of the second kind with a symmetric kernel, $K^{*}\left(\mathbf{x}_{0}, \mathbf{x}\right)$, by the following transformation:

$$
\begin{gathered}
B_{\lambda}^{*}(\mathbf{x})=B_{\lambda}(\mathbf{x})[1-\epsilon(\lambda, \mathbf{x})]^{1 / 2} \\
K^{*}\left(\mathbf{x}_{0}, \mathbf{x}\right)=[1-\epsilon(\lambda, \mathbf{x})]^{1 / 2}\left[1-\epsilon\left(\lambda, \mathbf{x}_{0}\right)\right]^{1 / 2} K\left(\mathbf{x}_{0}, \mathbf{x}\right) .
\end{gathered}
$$

The resulting equation is given below:

$B_{\lambda}^{*}(\mathbf{x})=\epsilon(\lambda, \mathbf{x})[1-\epsilon(\lambda, \mathbf{x})]^{-1} B_{b,{ }_{\lambda}}[T(\mathbf{x})]+[1-\epsilon(\lambda, \mathbf{x})]^{1 / 2} E_{\lambda,} e^{(\mathbf{x})}$

$$
+\int_{c} B_{\lambda}^{*}\left(\mathbf{x}_{0}\right) K^{*}\left(\mathbf{x}_{0}, \mathbf{x}\right) d A\left(\mathbf{x}_{0}\right)
$$


This transformation is interesting because it permits the use of certain theorems and methods introduced by Moon [12] even when $\epsilon(\lambda, \mathbf{x})$ is not constant.

\section{References}

[1] W. Wein and O. Lummer, Methode zur Prufung des Strahlungsgesetzes absolut schwarzer Korper, Annalen der Physik 56, 451-456 (1895).

[2] G. Ribaud, Traité de Pryométrie (Edition de la Revue d'Optique Theroique et Instrumentale), Paris, 1931, p. 231.

[3] A. Gouffé, Corrections d'ouverture des corps-noirs artificiels compte tenu des diffusions multiples internes, Rev. d'Optique, 24, No. 1-3, 1-10 (Jan.-Mar., 1945).

[4] M. Michaud, Facteur d'Emission des Cavités de Formes Geometriques Simples, Comptes Rendus 226, 999 (1948).

[5] C. H. Page, private communication.

[6] C. von Fragstein, On the formulation of Kirchhoff's Law and its use for a suitable definition of diffuse reflection factors, Translation from Optik, 12, No. 2,60-70 (1955), S.L.A. Translation Center.

[7] F. J. Kelly and D. G. Moore, A test of analytical expressions for the thermal emissivity of shallow cylindrical cavities, Applied Optics 4,31 (1965).

[8] E. M. Sparrow, L. U. Albers and E. R. G. Eckert, Thermal radiation characteristics of cylindrical enclosures, J. Heat Transfer, Trans. ASME, Series C, pp. 73-81 (Feb. 1962).

[9] E. M. Sparrow and L. U. Albers, Apparent emissivity and heat transfer in a long cylindrical hole, J. Heat Transfer, Trans. ASME, Series C, 82, 253-255 (1960).

[10] E. M. Sparrow and V. K. Jonsson, Absorption and emission characteristics of diffuse spherical enclosures, J. Heat Transfer, Trans. ASME, Series C, 84, No. 2, 188-189 (1962).

[11] E. M. Sparrow and V. K. Jonsson, Radiant emission characteristics of diffuse conical cavities, J. Opt. Soc. Am. 53, No. 7, 816-821 (July 1963).

[12] P. Moon, On interreflections, J. Opt. Soc. Am. pp. 195-205 (30 May 1940).

(Paper 69B3-148) 